\section{Sternal osteomyelitis after bacillus Calmette-Guérin vaccination}

\author{
Rolandas Selvestravičius, 1 \\ Elena Sučilienè,1 Kęstutis Saniukas, ${ }^{2}$ \\ Odeta Bobelytè,1 Vytautas Usonis1 \\ ${ }^{1}$ Clinic of Children's Diseases, ${ }^{2}$ Clinic of \\ Abdominal Surgery, Vilnius University, \\ Vilnius, Lithuania
}

\begin{abstract}
Presented here is the case of a nine-month-old boy with the osteomyelitis of the upper area sternum caused by bacillus Calmette-Guerin (BCG), the Danish 1331 strain vaccine against tuberculosis. Upon examination, a swelling of approximately $2 \times 3 \mathrm{~cm}$ diameter was observed in the upper sternal area. The mass was hard, fixed and sensitive to palpation with no local skin hyperaemia. Chest X-rays revealed a round mass anterior to the sternum, suggesting a diagnosis of osteomyelitis. A consequent sternal biopsy was performed and Mycobacterium bovis BCG was identified by a positive growth culture.
\end{abstract}

\section{Introduction}

The bacillus Calmette-Guerin (BCG) vaccination is globally used for the prevention of tuberculosis throughout the world. In Lithuania $97.7 \%$ of newborns were given BCG Danish 1331 strain vaccine in 2014.1 Although this vaccine prevents severe forms of tuberculosis (TB) and has a high safety profile, a variety of complications can sometimes develop. Mostly the complications are encountered in local skin complications, 2,3 regional suppurative lymphadenitis; ${ }^{4}$ however, it can also cause disseminated disease,, 5 a fatal prognosis in infants with a particular immune deficiency. Bone osteomyelitis is a relatively rare complication of the BCG vaccination and often is involving long bone epiphysis. 7,8 There are only a few reports about sternal osteomyelitis with an abscess. ${ }^{9}$

\section{Case Report}

A nine-month-old boy was admitted to Vilnius University Children's Hospital, Lithuania, due to a swelling mass of the anterior chest wall, which had appeared a week prior to hospitalization. He had fever, which had lasted several days a month before the appearance of the mass, but the boy was otherwise healthy and well-developed exhibiting sufficient weight gain. He received his BCG vaccination two days after his birth with no significant side effects after vaccination. At the age of three months he experienced a mild fall with no serious injuries. At the age of four months he had pneumonia, which was treated with a course of oral cefuroxime followed by a daily vibrant massage for five days.

Swelling of approximately $2 \times 3 \mathrm{~cm}$ diameter appeared in the upper sternal area at the age of nine months. The mass was hard, fixed and sensitive to palpation with no local skin hyperemia. No other signs of infection (e.g., fever) were present. Routine laboratory tests were within normal range. Frontal chest $X$-rays revealed a round mass anterior to the sternum, suggesting a diagnosis of osteomyelitis.

After performing surgical resection of the affected sternum, a histopathologic examination of the mass tissue showed active granulomatous inflammation, the granulomas contains macrophages, epithelioid cells and multinucleated giant cells (Langhans giant cells), with subchondral bone destruction and necrotic areas suggesting Mycobacterium tuberculosis etiology. However, no acid-resistant bacilli were found. A diagnosis of chronic osteomyelitis was defined with reference to the histopathologic findings.

After surgery the patient was consulted by many specialists and specific tests were performed for further elaboration of the diagnosis. There was not substantiation for neoplastic processes, therefore an oncological diagnosis wasn't performed. Normal immunologic tests (normal values of immunoglobulin A, M, G, Ag, normal phagocytic activity of neutrophils and nitro-blue tetrazolium test, a sufficient level of circulating immune complexes and negative immunofluorescence assay of Human Immunodeficiency Virus (HIV) ruled out a possible diagnosis of primary or secondary immunodeficiency, ruling out the possibility of hematological disease. Changes in the chest computer tomography (CT) scan revealed a round mass with central hypodense areas both with and without contrast (Figure 1). Additional data was collected from the patient's parents claiming that the boy's grandmother was suspected of suffering from tuberculosis. A consequent sternal biopsy was performed and $M$. bovis BCG was identified by a positive growth culture in liquid MGIT 960 system using the GenoType method (BD Biosciences, Sparks, MD, USA). These findings confirmed the diagnosis of M. tuberculosis BCG osteomyelitis. Treatment with rifampicin, isoniazid, ethambutol and streptomycin was started. Control CT scans were performed every 3 months. Full recovery was achieved after six months of antibacterial therapy (Figure 2).

\section{Discussion and Conclusions}

The BCG vaccine is the oldest vaccine still in
Correspondence: Vytautas Usonis, Vilnius University, Clinic of Children's Diseases. Santariškių 4, LT-08406 Vilnius, Lithuania. Tel.: +370.618.70749 - Fax: +370.5.272.0368. E-mail: vytautas.usonis@mf.vu.lt

Key words: BCG osteomyelitis; BCG vaccine; Adverse reactions; Tuberculosis.

Acknowledgments: the authors would like to thank all the colleagues who contributed to treatment of our patient and the family of our patient for their support.

Contributions: the authors contributed equally.

Conflict of interest: the authors declare no potential conflict of interest.

Received for publication: 30 May 2016.

Accepted for publication: 4 August 2016.

This work is licensed under a Creative Commons Attribution NonCommercial 4.0 License (CC BYNC 4.0).

(C) Copyright R. Selvestravičius et al., 2016 Licensee PAGEPress, Italy

Pediatric Reports 2016; 8:6626

doi:10.4081/pr.2016.6626

use and has been used since 1921.10,11 It is one of the most widely used vaccines globally. Currently the most commonly used vaccine strains are the following: Copenhagen strain 1331 (Danish 1331), Tokyo 172-1 and Russian BCG-I.12 In Lithuania, the Copenhagen strain 1331 (Danish 1331) containing the Danish 1331 strain is currently being used. This strain is known to induce more adverse reactions as compared with the other strains. WHO reference to the risk of adverse events has generally been considered to below. Mild adverse reactions such as papule at the injection site, ulceration, and scars are considered to be common for all BCG vaccines. Severe adverse reactions like lymphadenitis suppuration occur in one case per $1000-10,000$ vaccinations. Other severe adverse reactions are extremely rare: disseminated BCG, one per $230,000-640,000$ vaccinations; immune reconstitution syndrome, one per 640,000 ; osteitis, one case per 3.3-10;8 systemic cutaneous skin lesions, a single case reports only. ${ }^{13}$ In Lithuania about 30,000 BCG vaccinations are performed every year and severe adverse reactions following BCG vaccination of newborns during 2005-2015 period were as follows: severe local skin complications, 8 cases; osteitis, 6 cases; lymphadenitis, 287 cases, disseminated BCG, 4 cases. [(Severe adverse events following BCG vaccination of newborns in Lithuania. SVEIDRA (Compulsory Health Insurance Fund information system). 2005-2015 period. Unpublished data.)]

Tuberculous osteomyelitis is usually the result 
of a hematogenous dissemination of BCG strains due to the abundant blood supply at the time of the initial bacteremia.14 Sometimes, tubercle bacilli might be transported to the sternum via the blood following the development of the primary pulmonary lesion. Osteomyelitis of the sternum usually appears as a complication of chest trauma also sternotomy, mediastinitis, or a subclavian intravenous line insertion. 15 In our case it is not clear which factor influenced the BCG sternal osteomyelitis. Our patient had a mild fall at the age of three months with no serious injuries; also, at the age of four months he suffered from pneumonia. In addition to the treatment with oral cefuroxime he received daily vibrant massages, which might have influenced the $M$. bovis trans-

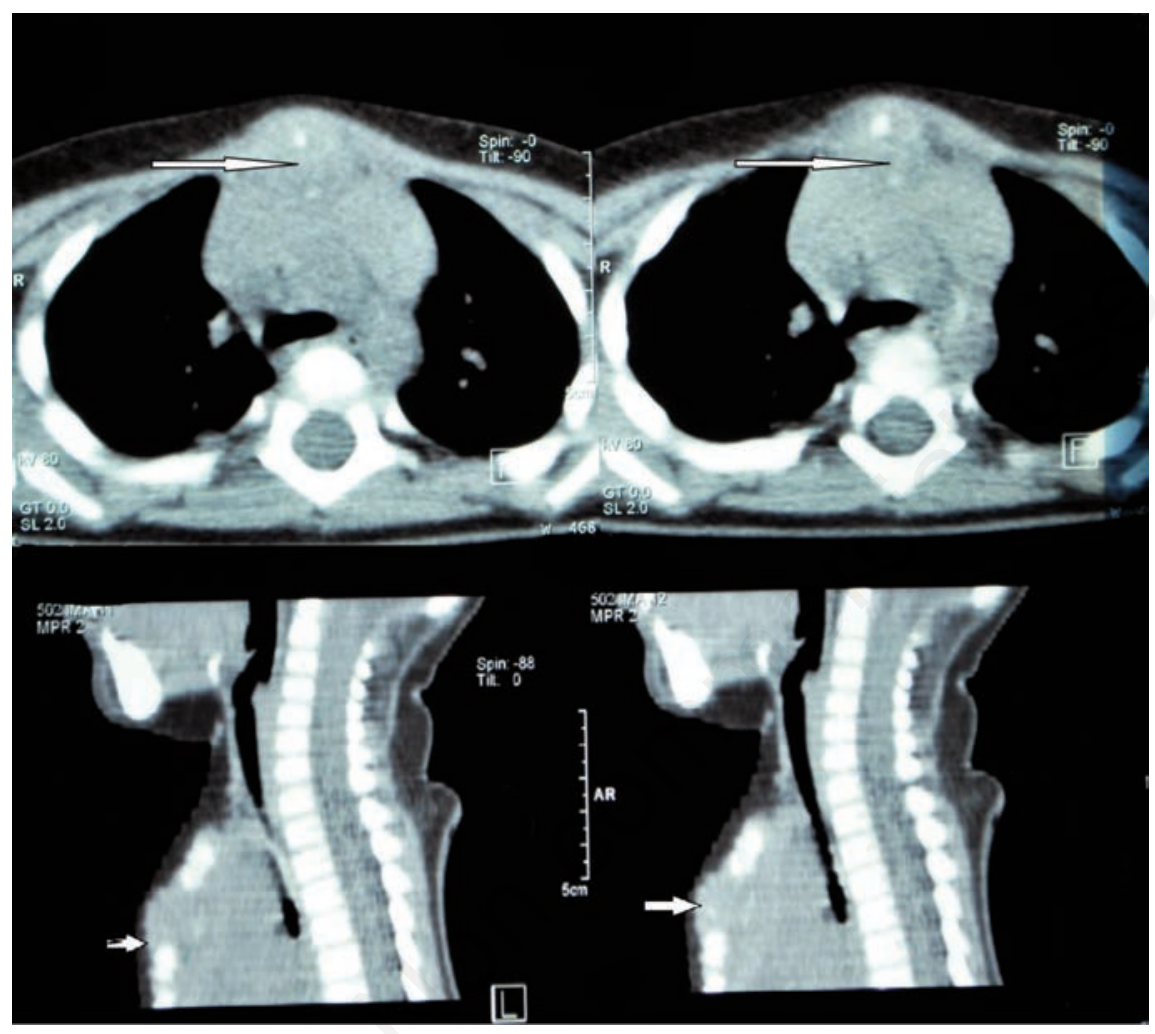

Figure 1. Computed tomography without contrast-enhanced axial and multi planar reconstruction sagittal images show the mass in the anterior mediastinum, around the manubrium of sternum.

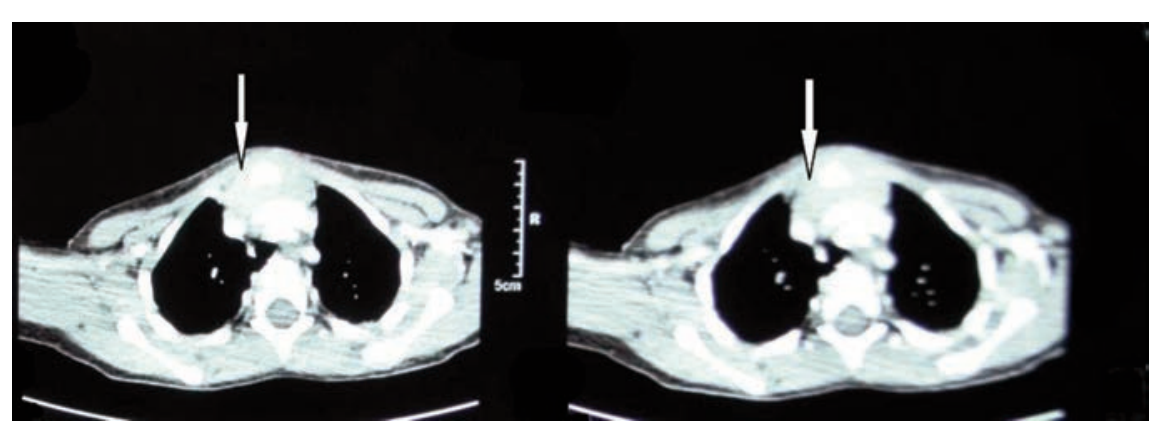

Figure 2. Axial contrast-enhanced computed tomography showing that the mass is reducing around sternum.

portation to the sternum via the blood. Our patient completely recovered after six months of complex TBC specific antibacterial therapy. The follow up during eight years confirmed that stable recovery has been achieved.

The case reported is a rare complication of BCG vaccination requiring surgical intervention and specific treatment. Although cases of BCG osteomyelitis of long bones are reported as known adverse event after BCG vaccination, involvement of sternum is extremely rare and might be of interest. 